\title{
Mental Health Status of Infertile Women in Bangladesh
}

\author{
Asoke Kumar Saha* , Kazi Saifuddin, Fatema-Tu-Zohra Binte Zaman, Nishat Jahan Nisha \\ Department of Psychology, Jagannath University, Bangladesh
}

Copyright $(2015$ Horizon Research Publishing All rights reserved.

\begin{abstract}
Special attention has been paid to psychological health of infertile women during the last few years (Domar [1]. Therefore, the present study was conducted to find out the differences in the mental health among fertile and infertile women. The study is conducted to come across the level of difference in mental health between fertile and infertile women. In order to measure the dependent variable, the investigators used Bengali version of Goldberg's mental health scale. A simple t-test was used to analysis the data collected from 50 respondents. The results revealed that infertile women have lower mental health score than fertile women which proved the formulated hypothesis. The results are also discussed in the context of personal mental health and happiness in the social paradigm of Bangladesh.
\end{abstract}

Keywords Mental Health, Infertile, Fertile, Women

\section{Introduction}

Mental health complications of childless marriages have been documented in the developed (Matsubayashi et al. [2], Monga et al., [3], King [4], Tan et al. [5], Andrews et al. [6], Ulbrich et al. [7], and less developed nations of the world (Sami and Ali [8], Dyer et al., [9], Fiander [10], Jindal \& Dhall [11]. Infertility is an important area of clinical work that has continued to stimulate a significant degree of worldwide research interest. Infertility may lead to physical, emotional and financial burden (Monga et al., [03]. Infertility is doubtlessly a severe distressful experience for many infertile couples. Freeman et al., [12] reported that $50 \%$ of couples considered infertility as the most disappointing experience in their lives. Another study performed by Mahlstedt et al., [13] showed that $80 \%$ of infertile couples described infertility as a stressful or very stressful experience.

Mental Health indicates a situation if a person can adjust with their social demands and stress. It describes a level of psychological wellbeing or an absence of mental disorder from the perspective of positive psychology. According to the World Health Organization (WHO, http://www.who.int/mental_health/en/ [14]) mental health is a state of well-being in which the individual realizes his or her own abilities can cope with the normal stresses of life, can work productively and fruitfully and is able to make a contribution to his or her community. Mental Health is an emotional, behavioral and social maturity or normalcy, the absence of a mental or behavioral disorder, a state of psychological well-being in which one has achieved a satisfactory integration of one's instinctual drives acceptable to both oneself and one's social milieu, an appropriate balance of love, work and leisure pursuits.

Fertility refers to the condition, quality, or degree of being fertile. It is the ability to produce offspring or the power of reproduction. Fertility is the natural capability to produce offspring. Human fertility depends on factors of nutrition, sexual behavior, culture, instinct, endocrinology, timing, economics, way of life, and emotions. A woman's fertility usually peaks in her early 20 s, and drops considerably after age 35. Menopause typically occurs during a women's midlife (usually between ages 45 and 55). This is considered the end of the fertile phase of a woman's life. The use of fertility drugs and/or in-vitro-fertilization can increase the chances of becoming pregnant at a later age. Successful pregnancies facilitated by fertility treatment have been documented in women as old as 67 (WHO http://www.who.int/mental_health/en/ [14].

Infertility primarily refers to the biological inability of a person to contribute to conception; a lack of fertility is called infertility. Infertility may also refer to the state of a woman who is unable to carry a pregnancy to full term. Definitions of infertility differ, with demographers tending to define infertility as childlessness in a population of women of reproductive age. Infertility can be divided into two types: primary and secondary. Couples with primary infertility have never been able to conceive, on the other hand, secondary infertility is difficulty conceiving after already having conceived. There are some common causes of infertility which are given as follows- Female causes, Male causes, combined causes, unexplained causes and others.

According to Brigitte Baldur-Feskov (Danish Cancer Society Research Center, Copenhagen) and colleagues, who analyzed data from 98,737 Danish women who had seen a doctor about infertility between 1973 and 2003. Of all the women analyzed, 4677 were hospitalized with mental health problems. Of these, 2507 had not been successful in producing a child, compared with 2107 women who had 
successful fertility treatment that resulted in at least one child. The team also noted that women who did not go on to have children were 47 per cent more likely to be hospitalized for schizophrenia than those who gave birth (Tiffany O'Callaghand [15].

Other researchers found that couples experience stigma, sense of loss, and diminished self-esteem in the setting of their infertility. It is seen in general, in infertile couples women show higher levels of distress than their male partners (Wright [14]. A number of studies have found that the incidence of depression in infertile couples presenting for infertility treatment is significantly higher than in fertile controls. Anxiety has also been shown to be significantly higher in infertile couples when compared to the general population. A study conducted on 10 depressed and 13 normal women suggests that depression is associated with abnormal regulation of luteinizing hormone, a hormone that regulates ovulation. Special attention has been paid to psychological health of infertile women during the last few years. Therefore, the present study was undertaken with a view to highlight the differences in the mental health conditions between fertile and infertile women in the context of Bangladesh as the infertility is found to be a major social problem which associated to individuals health and wellbeing.

\section{Rationale of the Study}

Special attention has been paid to psychological health of infertile women during the last few years (Domar [1]. There are common depression in infertile couples presenting for infertility treatment is significantly higher than in fertile controls. It is often said that a sound mind resides in a sound body. If a person is physically not healthy it is very usual that the person will go through some mental health problems. The idea of the present study was conceived to compute the mental health level of infertile women which is obvious. There is no such study that has been conducted so far specifically on infertile women in relation to mental health. This study also aimed to detect the prevention method and remedial help to such women if any. Thus, the present study was designed considering the importance to know the mental health of infertile women as fertile women and to compare the same.

\section{Objective of the study}

The major objective of this study is to find out the mental health status of infertile and fertile women in Dhaka City and to compare the mental health status between these groups. Because mental health of infertile women is considered a very significant variable associated with the well-being of the infertile women is almost every culture.

\section{Hypothesis}

The major hypothesis was Infertile women will have more mental health problem than fertile women, thus fertile women would show better mental health than infertile women.

\section{Methods}

\section{Design}

The present study used having children or not, background as independent variables and mental health problem as dependent variable. Thus, the study used two groups to examine deference of mental health of fertile and infertile women using t-test analysis.

\section{Sample}

Fifty (50) participants were selected purposively from Dhaka the capital city of Bangladesh from the following four health care institutions i.e. Infertile Care \& Research Centre (ICRC), Bangladesh Infertile Care Centre, and Hope \& Care Hospital. Participants were equally divided into infertile women $(n=25)$ and fertile women $(n=25)$. The respondents' marital duration was 5 years and above for the fertile and infertile women. The educational levels of the participants were secondary school certificate passed. Average infertile period of the respondent was recorded as 7.5 years and duration of fertility for fertile women was 8 years from the first delivery. The sample size is relatively small considering difficulties to get infertile women and less willing to participate in the study.

\section{Measures}

The present study required the following questionnaire and a demographic form: 1. Goldberg's Mental Health Scale to discern mental health; and 2. The Personal Information Form (PIF) for demographic information, and Each measure has been described in details herein:-

1. Goldberg's Mental Health Scale: Bangla translation of Mental Health Scale of Goldberg [17] was used in the present study. It is a 12 item self-report questionnaire used for assessing mental health of fertile and infertile women. This self-report instrument contains six positive and six negative items. Responses were given weights of $0,1,2$, and 3 . Items were answered on a 4 point response format ("Not at all", "Somewhat", "To a considerable extend" and "To a great extend"). Positive items were scored in 4 points, from 3 to 0 and the negative in the reverse order from 0 to 3 . Total scores are the sum of all the items, with a range of 0 to 36 . High score in the scale indicates the high mental health problems. The reliability of the Bengali version of the scale of Sarker and Rahman, [18] was measured by parallel form method which was found to be quite satisfactory $(\mathrm{r}=0.69)$.

2. The Personal Information Form (PIF): The PIF elicited demographic, personal, and social information which need to be collected for verification of the finding if desired results.

\section{Procedure}

The investigators formed two groups and individually approached each participant and asked them about their 
infertility and fertility. Participants who identified themselves as infertile were taken as respondents for the study. Consent from the women was taken and the data was collected with the help of doctors and gynecologists. Women were encouraged to ask any question that came into mind regarding the questionnaire. To conduct the study 50 participants were equally divided into fertile and infertile group. Upon initial meeting, each participant was informed about the confidentiality regarding information collected from them. There was no time limit for completing the questionnaire but the respondents completed it within 10 to 20 minutes. Many women were hesitant to rate different personal matter. A good rapport was built to get correct responses and some necessary instructions and guidelines were provided to them for properly filling the questionnaire. They were requested to fill the questionnaire as per the instructions and after completion they returned the questionnaire and were thanked for their participation and cooperation.

\section{Results}

The aim of the present investigation is to find out the level of mental health of infertile and fertile women in Bangladesh. The data obtained from the returned surveys were analyzed and responses to prove the hypothesis were made using descriptive statistics, including item means, standard deviations and $t$-test. The obtained results are presented in table 1. All statistical analyses were carried out using the statistical program SPSS version 16.0 for windows with the significance level of 0.05 .

Table 1. Differences of mental health between infertile and fertile women

\begin{tabular}{lcccc}
\hline Groups & $\mathrm{M}$ & $\mathrm{SD}$ & t-value & Sig. level \\
\hline Infertile & 22.80 & 5.78 & $5.50 *$ & 0.05 \\
Fertile & 14.24 & 5.20 & & \\
\hline$* p<0.05$ & & & &
\end{tabular}

The result presented in Table-1 indicates that there is significant difference in mental health between infertile and fertile women $(\mathrm{t}=5.50, p<.05$, Infertile Mean $=22.80, \mathrm{SD}=$ 5.78 , Fertile Mean $=14.24, \mathrm{SD}=5.20$ ). The findings further indicate that infertile women obtained higher scores on mental health problems than fertile women and the difference is statistically significant. Thus, the results confirmed the hypothesis formulated in the study.

\section{Discussion}

The present study was an attempt to explore the phenomenon of differential mental health status of infertile and fertile women. The unequal mental health status between infertile and fertile women have direct relevant. The study was conducted primarily to find differences in mental health problem in order to construct the purpose of study using the relevant questionnaire Goldberg [17] Mental Health Scale.
The result presented in Table-1 indicates that there is significant difference in mental health between infertile and fertile women $(t=5.50, p<.05)$. The findings further indicate that infertile women obtained higher scores on mental health problems than fertile women and the difference is significant.

The results of the present studies also lend support that mental health complications of childless marriages have been documented in several studies i.e. Matsubayashi et al. [2], Monga et al. [3], King [4], Tan et al., [5], Andrews et al. [6], Ulbrich et al. [7], and less developed nations of the world (Sami and Ali [8], Dyer et al., [9], Fiander [10], Jindal \& Dhall [11]. Thus, the findings provide confirmation to the hypothesis i.e. infertile women will have more mental health problems than fertile women. It was also found that regardless of having children backgrounds the fertile women participants expressed significantly higher feelings of satisfaction and infertile women participants expressed significantly higher feelings of depression. A very large section of women in our society suffer from these problems which are also supported by the findings of Domar [1]. However, it is not a single problem. In fact it is a multipart problem because it creates various social problems such as second marriage, divorce, extra marital affairs, endeavors and sometimes successful attempts of stealing children. For this reason, it is necessary to know the details of infertile women's mental health problem and find out the prevention methods from these problems.

There are certain limitations of the study. It is insufficient to explore all the aspects of mental health problems. To understand the phenomenon of mental health problem of Bangladeshi women it is necessary to utilize multi-dimensional factors and it needs extensive empirical verification. One of the limitations is that the question type was so personal many participants were nonchalant to provide information correctly. Another limitation is that the sample was very small. This study contributes to develop the mental health state of infertile women and help them overcome these problems.

\section{REFERENCES}

[1] Domar, A.D., Broome, A., Zuttermeister, P.C., Scibel M, \& Friedman, R. (1992). The prevail and predictability of depression in infertile women. Investing in mental health World Health Organization. Fertile Steril, 58, 1158-1163.

[2] Matsubayashi, H., Hosaka, T., \& Izumi, S. (2004). Increased depression and anxiety in infertile Japanese women resulting from lack of husband's support and feelings of stress. General Hospital Psychiatry, 26, 398-404.

[3] Monga, M., Alexandrescu, B., \& Katz, S.E. (2004). Impact of infertility on quality of life, marital adjustment, and sexual function. Urology, 63, 126-130.

[4] King, R.B. (2003). Sub fecundity and anxiety in a nationally representative sample. Social Medicine, 56,739-751. 
[5] Tan, S., Duan, X., \& Tang, X. (2001). The psychological effect of clinical examination on women with tubal infertility. Hua Xi Yi Ke Da Xue Xue Bao. 32, 254-256.

[6] Andrews, F.M., Abbey, A., \& Halman, L.J. (1991). Stress from infertility, marriage factors, and subjective well-being of wives and husbands. Journal of Health and Social Behaviour, 32, 238-53.

[7] Ulbrich, P.M., Coyle, A.T., \& Llabre, M.M. (1990). Involuntary childlessness and marital adjustment: his and hers. Journal of Sex Marital Therapy, 16,147-158.

[8] Sami, N., \& Ali, T.S. (2006). Psychosocial consequences of secondary infertility in Karachi. Journal of Pakistan Medical Association, 56, 19-22.

[9] Dyer, S.J., Abrahams, N., \& Mokoena, N.E. (2005). Psychological distress among women suffering from couple infertility in South Africa: a quantitative assessment. Human Reproduction, 20, 1938-1943.

[10] Fiander, A. (1991). Infertility: an approach to management in a district hospital in Ghana. Trop Doct, 21, 36-37.

[11] Jindal, U.N., \& Dhall, G.I. (1990). Psychosexual problems of infertile women in India. International Journal of Fertility, $35,222-225$
[12] Freeman, E.W., Rickels, K., Tausig, J., Boxer, A., Mastroianni, L., \& Tureck, R.W. (1987). Emotional and psychosocial factors in follow-up of women after IVFET treatment. A pilot investigation. Acta Obstet Gynecol Scand, $66,517-521$.

[13] Mahlstedt, P.P., Macduff, S., \& Bernstein, J. (1987). Emotional factors and the in vitro fertilization and embryo transfer process. Journal of In Vitro Fertility Embryo Transfer, 4, 232-236.

[14] WHO: http://www.who.int/mental_health/en/

[15] Tiffany O'Callaghand (2012). Infertility may increase risk of mental disorder.

[16] Wright, J., Duchesne, C., Sabourin, S., Bissonnette, F., Benoit, J., Girard, Y. (1991). Psychosocial distress and infertility: men and women respond differently. Fertility Steril, 55, 100-108.

[17] Goldberg, D.P. (1972). The detection of psychiatric illness by questionnaire. Oxford University Press, London.

[18] Sarker, N.R. \& Rahman, A. (1989). Occupational stress and mental Health of working environment. UFG, Dhaka. 